\title{
Symposium on Ultrafast Electron Microscopy and Ultrafast Science
}

Dynamic characterization techniques have been utilized in the fields of biology, chemistry, physics, and materials science for many years. Techniques range from neutron scattering to X-ray diffraction. Two of the fields experiencing much development recently have been electron-based techniques. Namely, ultrafast electron diffraction (UED) and ultrafast electron microscopy (UEM) have been advancing rapidly, but unfortunately, in parallel. We are approaching an era where the convergence of these two techniques could open up a wide range of scientific and technological opportunities and advancements.

Recent instrumentation development thrusts have focused on increased resolution, both spatially and temporally. The applications include aberration correction, both Cc and Cs, in transmission electron microscopy (TEM), stroboscopic and pump-probe photoemission TEM, and, finally, advances in femtosecond-scale electron diffraction techniques. Users of these techniques could all benefit from one another in a complimentary manner. We look to combine existing knowledge of the developing instrumentation to look to the future for a possible solution to a myriad of scientific problems.

A symposium, entitled "Ultrafast Electron Microscopy and Ultrafast Science," was organized for the 2008 Microscopy and Microanalysis (M\&M) Annual Meeting held in August in Albuquerque, NM. The goal of this symposium was to highlight results from the various research programs under way across the world, and to bring together experts from the microscopy and ultrafast diffraction community to share new ideas. This is the third symposium of its type, following those held at the annual M\&M meetings in Hawaii and Chicago. The following six papers of this month's issue of Microscopy and Microanalysis were presented during this symposium.

Guest Editors

Mitra L. Taheri, Drexel University

Nigel D. Browning, University California-Davis and Lawrence Livermore National Laboratory

John Lewellen, Naval Postgraduate School/ Advanced Proton Source 